\title{
2. Dokumentation
}

\section{Otmar Jung}

a) Daten zu Volksbegehren und Volksentscheid

Thüringen, 20. März bis 19. Juli 2008

Volksbegehren „Mehr Demokratie in Thüringer Kommunen“

\begin{tabular}{l|lll}
\hline Stimmberechtigte & gültige Eintragungen & in $\%$ & Quorum \\
\hline 1.960 .053 & 235.530 & 12,02 & 10 \\
\hline
\end{tabular}

Zahl der gültigen Eintragungen und Prozentsatz nach Pressemitteilung der Landtagspräsidentin v. 23.10.2008, http://www.thueringen.de/tlt/aktuell/presse/ 36049/ index. asp (Zugriff 9.6.2009). Das 10-Prozent-Quorum galt, weil die Initiatoren die freie Sammlung gewählt hatten. - Das Thüringer Landesamt für Statistik ist laut Auskunft v. 23.6.2009 mit dem Volksbegehren nicht befaßt worden. Auf der Internet-Homepage des „Bündnisses für Mehr Demokratie in Thüringen“ (http:// www.thueringen.mehr-demokratie.de/volksbegehren-erfolgreich.html, Zugriff 3.7.2009) finden sich Ergebnisse sowohl für die kreisfreien Städte und die Landkreise als auch für die Gemeinden: Abschlussuebersicht_Volksbegehren_Kreise.pdf und Abschlussuebersicht_Volksbegehren_Gemeinden.pdf. Allerdings sind dort nur die gesammelten und nicht die als gültig anerkannten Unterschriften aufgeführt. Gleichwohl geben diese Übersichten einen gewissen Aufschluß über die kleinräumige Verteilung. - Ergebnis: Das Volksbegehren ist zustande gekommen.

Hamburg, 19. September bis 9. Oktober 2008

Volksbegehren ,Eine Schule für Alle“

\begin{tabular}{l|lll}
\hline Stimmberechtigte & vorgelegte Unterschriften & in \% & Quorum \\
\hline 1.236 .671 & 51.509 & & $61.834=5 \%$ \\
\hline
\end{tabular}

Zahlen nach Mitteilung des Senats an die Bürgerschaft, Bürgerschaft Drs. 19/1441 v. 4.11.2008, http://www.buergerschaft-hh.de/parldok/ (Zugriff 9.6.2009). Von einer Überprüfung der Gültigkeit der Eintragungen wurde abgesehen, da sie für das Ergebnis ohne Bedeutung war. - Nach Auskunft des Statistischen Amtes für Hamburg und Schleswig-Holstein v. 25.6.2009 werden die Ergebnisse von Volksbegehren nicht statistisch aufbereitet bzw. ausgewertet. - Ergebnis: Das Volksbegehren ist nicht zustande gekommen. 
Brandenburg, 10. Oktober 2008 bis 9. Februar 2009

Volksbegehren „Keine neuen Tagebaue - für eine zukunftsfähige Energiepolitik“

\begin{tabular}{l|lll}
\hline Stimmberechtigte & gültige Eintragungen & in \% & Quorum \\
\hline 2.134 .234 & 24.501 & $1,15 \%$ & $80.000=3,75 \%$ \\
\hline
\end{tabular}

Zahl der gültigen Eintragungen nach Bek. des Gesamtergebnisses v. 18.2.2009, GVBl. I S. 14; Zahl der Stimmberechtigten nach Mitteilung des Landesabstimmungsleiters v. 16.2.2009, http://www.wahlen.brandenburg.de/cms/detail.php/bbl. c.144876.de (Zugriff 17.2.2009), dort auch kleinräumige Aufgliederung nach kreisfreien Städten und Landkreisen. Prozentwerte eigene Berechnung. - Ergebnis: Das Volksbegehren ist nicht zustande gekommen.

Berlin, 22. September 2008 bis 21. Januar 2009

Volksbegehren ,,Wir wollen Wahlfreiheit! Für die Einführung des Wahlpflichtbereichs Ethik/Religion! " (Pro Reli)

\begin{tabular}{l|lll}
\hline Stimmberechtigte & gültige Eintragungen & in $\%$ & Quorum \\
\hline 2.441 .496 & 265.823 & $10,9 \%$ & $7 \%$ \\
\hline
\end{tabular}

Bek. des Endgültigen Ergebnisses v. 4.2.2009, AB1. S. 366. Vgl. Pressemitteilung des Landesabstimmungsleiters v. 4.2.2009 (mit Aufgliederung nach Bezirken) http://www.wahlen-berlin.de/wahlen/framesets/vb-2008.htm (Zugriff 5.2.2009). Ergebnis: Das Volksbegehren ist zustande gekommen.

Hamburg, 23. Januar bis 12. Februar 2009

Volksbegehren „,Mehr Demokratie - Ein faires Wahlrecht für Hamburg “

\begin{tabular}{l|lll}
\hline Stimmberechtigte & gültige Unterschriften & in \% & Quorum \\
\hline 1.236 .671 & (mindestens) 62.310 & & $61.834=5 \%$ \\
\hline
\end{tabular}

Zahlen nach Mitteilung des Senats an die Bürgerschaft, Bürgerschaft Drs. 19/2534 v. 10.3.2009, http://www.buergerschaft-hh.de/parldok/ (Zugriff 9.6.2009). Nach Ermittlung von 62.310 gültigen Eintragungen wurde die weitere Überprüfung der insgesamt abgegebenen 76.086 Unterschriften eingestellt. - Ergebnis: Das Volksbegehren ist zustande gekommen. 
Berlin, 26. April 2009

Volksentscheid ,über die Einführung des Wahlpflichtbereichs Ethik/Religion“

\begin{tabular}{l|llllllll}
\hline $\begin{array}{l}\text { Stimmberech- } \\
\text { tigte }\end{array}$ & $\begin{array}{l}\text { Abgegebene } \\
\text { Stimmen }\end{array}$ & in \% & ungültig & gültig & Ja & in \% & Nein & in \% \\
\hline $\mathbf{2 . 4 4 5 . 6 9 9}$ & 713.095 & 29,2 & 1.370 & 711.725 & 345.004 & 48,5 & 366.721 & 51,5 \\
\hline
\end{tabular}

Absolute Zahlen (außer „gültig") nach Bek. des Endgültigen Ergebnisses v. 5.5.2009, ABl. S. 1186. Vgl. Bericht des Landesabstimmungsleiters: Volksentscheid über die Einführung des Wahlpflichtbereichs Ethik/Religion am 26. April 2009. Endg. Ergebnis, zugleich Statistischer Bericht B VII 4-1 (mit Aufgliederung nach Bezirken), http://www.wahlen-berlin.de/wahlen/framesets/ve-2009.htm (Zugriff 9.6.2009). - Prozentwerte für „Ja“ und „Nein“ eigene Berechnung, da die amtlichen Veröffentlichungen auf die abgegebenen, anstatt auf die abgegebenen gültigen Stimmen prozentuieren. - Ergebnis: Der volksbegehrte Gesetzentwurf ist „echt“ gescheitert.

Berlin, 26. Januar bis 25. Mai 2009

Volksbegehren ,, Wahlfreiheit für Wirte und Gäste - kein Rauchverbot in Berliner Gaststätten “

\begin{tabular}{l|lll}
\hline Stimmberechtigte & gültige Eintragungen & in $\%$ & Quorum \\
\hline 2.446 .034 & 61.644 & 2,5 & $7 \%$ \\
\hline
\end{tabular}

Bek. des Endgültigen Ergebnisses v. 9.6.2009, ABl. S. 1378; Pressemitteilung des Landesabstimmungsleiters v. 9.6.2009 (mit Aufgliederung nach Bezirken), http:// www.wahlen-berlin.de/wahlen/framesets/vb-2009.htm (Zugriff 9.6.2009). Ergebnis: Das Volksbegehren ist nicht zustande gekommen.

b) Daten zu Bürgerbegehren und Bürgerentscheid (Auswahl)

Bürgerentscheid in Berlin-Mitte, 28. September 2008

„, Gegen die Ausweitung der Parkraumbewirtschaftung in Berlin-Mitte“

\begin{tabular}{l|llllllll}
\hline $\begin{array}{l}\text { Stimmberech- } \\
\text { tigte }\end{array}$ & $\begin{array}{l}\text { Abgegebene } \\
\text { Stimmen }\end{array}$ & in \% & ungültig & gültig & Ja & in \% & Nein & in \% \\
\hline $\mathbf{2 1 4 . 8 7 3}$ & 25.077 & 11,7 & 82 & 24.995 & 19.878 & 79,5 & 5.117 & 20,5 \\
\hline
\end{tabular}

Absolute Zahlen nach der Veröffentlichung des Bezirksamtes unter http:// www.berlin.de/ imperia/ md/ content/ bamitte/ buergerdienste/ endg_ltiges_ergebnis.pdf (Zugriff 9.6.2009), mit kleinräumiger Aufgliederung nach Stimmbezirken. 
Das Ergebnis des Bürgerentscheids wurde (offenbar aus Versehen) bis Ende Juni 2009 noch nicht im „Amtsblatt für Berlin“ bekanntgemacht. - Prozentwerte eigene Berechnung. - Ergebnis: Da das Beteiligungsquorum von 15 Prozent verfehlt wurde, war der Bürgerentscheid nicht erfolgreich.

Bürgerentscheid in Berlin-Tempelhof-Schöneberg, 7. Juni 2009

„Das Denkmal Flughafen Tempelhof erhalten - als Weltkulturerbe schützen“

\begin{tabular}{l|lccccccc}
\hline $\begin{array}{l}\text { Stimmbe- } \\
\text { rechtigte }\end{array}$ & $\begin{array}{l}\text { Abgegebene } \\
\text { Stimmen }\end{array}$ & in \% & ungültig & gültig & Ja & in \% & Nein & in \% \\
\hline $\mathbf{2 5 0 . 8 3 9}$ & 95.111 & 37,9 & 3.528 & 91.583 & 62.053 & 67,8 & 29.530 & 32,2 \\
\hline
\end{tabular}

Bek. des Endgültigen Ergebnisses v. 11.6.2009, ABl. S. 1745. - Prozentwerte eigene Berechnung. - Ergebnis: Da das Beteiligungsquorum von 15 Prozent erreicht und die Vorlage mehrheitlich angenommen wurde, war der Bürgerentscheid erfolgreich. 